\title{
Subthalamic Nucleus-Deep Brain Stimulation Improves Autonomic Dysfunction in Parkinson's Disease
}

\author{
Feng Zhang \\ the First Hospital of Hebei Medical University \\ Feng Wang \\ Zhejiang University School of Medicine \\ Cong-Hui Li \\ the First Hospital of Hebei Medical University \\ Ji-Wei Wang \\ the First Hospital of Hebei Medical University \\ Chun-Lei Han \\ Capital Medical University \\ Shi-Ying Fan \\ Capital Medical University \\ Dong-Mei Gao \\ Capital Medical University

\section{Yu-Jing Xing} \\ the First Hospital of Hebei Medical University \\ Chen Yang \\ the First Hospital of Hebei Medical University \\ Jian-Guo Zhang \\ Capital Medical University \\ Fan-Gang Meng ( $\sim$ fgmeng@ccmu.edu.cn ) \\ Chinese Institute for Brain Research
}

\section{Research Article}

Keywords: Parkinson's disease, deep brain stimulation, subthalamic nucleus, autonomic dysfunction, non-motor symptoms

Posted Date: November 29th, 2021

DOI: https://doi.org/10.21203/rs.3.rs-1070327/v1

License: (c) (i) This work is licensed under a Creative Commons Attribution 4.0 International License. Read Full License

Version of Record: A version of this preprint was published at BMC Neurology on March 31st, 2022. See the published version at https://doi.org/10.1186/s12883-022-02651-z. 


\section{Abstract \\ Background}

To study the effects of subthalamic nucleus deep brain stimulation (STN-DBS) on autonomic dysfunction in Parkinson's disease (PD) patients.

\section{Methods}

57 PD patients, who underwent bilateral STN-DBS from March to December 2018, were retrospectively analyzed, preplanned assessments at baseline and postoperatively at 1, 3 and 6 months also included the Scales for Outcomes in Parkinson's Disease-Autonomic questionnaire (SCOPA-Aut), the Unified Parkinson's Disease Rating Scale (UPDRS) III score, levodopa equivalent day dose (LEDD), Parkinson's Disease Quality of Life Scale (PDQ-39), the Hamilton Anxiety Rating Scale (HAMA), the Hamilton Depression Rating Scale (HAMD).

\section{Results}

The SCOPA-Aut scores improved significantly [14.59\% (18.32\%), 24.00\% (27.05\%), 22.16\% (27.07\%), respectively, all $P$ $<0.001$ ] at 1 months, 3 months, 6months of STN-DBS respectively. Analysis of the SCOPA-Aut subitems showed significant improvement only in urine and thermoregulation subitems at 6 months after operation $(P<0.001)$. There was no significant correlation between the improvement rate of SCOPA-Aut scores and the improvement rate of PDQ-39 scores $(P>0.05)$ at 6 months after operation. SCOPA-Aut scores was positively correlated with age $(r=0.428, P=0.001)$; The improvement rate of SCCOPA-Aut scores was positively correlated with the improvement rate of HAMA and HAMD scores (HAMA: $r=0.325, P=0.015$; HAMD: $r=0.265, P=0.049$ ) at 6 months after operation.

\section{Conclusion}

STN-DBS can improve autonomic dysfunction symptoms of PD patients, urinary and thermoregulatory subitems of autonomic dysfunction were improved in the short term after operation. There was a close relationship between improved autonomic symptoms and improved anxiety and depression 6 months after operation. We should pay more attention to the autonomic dysfunction in Parkinson's disease, detailed preoperative evaluation and postoperative follow-up, so as to better improve the QOL of patients.

\section{Background}

PD is a common neurodegenerative disease of the central nervous system. Deep brain stimulation (DBS) is an effective treatment in advanced PD patients [1]. Subthalamic nucleus (STN) DBS has been shown to improve motor symptoms and quality of life (QOL) [2-4], whereas the effects on non-motor symptoms (NMS) have been less reported. NMS may have a greater impact on QOL than motor symptoms. Dysfunctions of the autonomic nervous system (gastrointestinal symptoms, urinary symptoms, cardiovascular symptoms, thermoregulation, pupillomotor function, sexual function) are common in PD, autonomic dysfunction may appear earlier than major motor symptoms of PD and significantly impair the quality of life. Autonomic dysfunction are associated with the accumulation of lewy bodies in the nervous system.

Parkinson's disease progression and dopaminergic drug therapy may also aggravate autonomic dysfunction. The effect of STN-DBS on autonomic symptoms such as sweating, urgency, increased frequency or incontinence, has not been well studied. The loss of central dopamine leads to motor symptoms, which can also lead to autonomic dysfunction, therefore, we speculated that STN-DBS may improve autonomic dysfunction. we have evaluated the impact of bilateral STN-DBS on 
autonomic dysfunction during 6 months of chronic stimulation and explored their relationship to motor symptoms, anxiety and depression and quality of life outcomes.

\section{Materials And Methods}

Between March and December 2018, a total of 57 subjects were hospitalized at the Department of Neurosurgery of the Beijing Tiantan Hospital affiliated to Capital Medical University, the First Hospital of Hebei Medical University and General Hospital of Ningxia Medical University for optimizing a previously performed STN-DBS, As mentioned in our previous article [5].

The study was approved by the Medical Ethics Committee of the First Hospital of Hebei Medical University and the Medical Ethics Committee of Beijing Tiantan Hospital affiliated to Capital Medical University, the ethical principles involved in this research strictly in accordance with the "Declaration of Helsinki" and all patients provided written informed consent, As mentioned in our previous article [5].

\section{Patient's Selection}

Evaluations were executed by neurologists specialized in movement disorders. Patients with advanced idiopathic PD diagnosed based on the diagnostic criteria for PD in China (2016 edition) and PD surgical treatment evaluation criteria [6, 7]. None of the patients had serious cognitive impairment or mental illness. All patients underwent preoperative testing and analyzed the levodopa challenge test (LCT), confirming that levodopa response needs to be improved by at least $30 \%$, and those who had complete imaging and scoring data and could follow up regularly. Morphologic MRI is performed to exclude patients with severe cerebral atrophy, ischemic disease.

\section{Clinical evaluation}

Demographic characteristics (age, gender, age at onset, duration of the disease) and disease severity, assessed by the Unified Parkinson's Disease Rating Scale (UPDRS)- $\bigotimes$ scores (range 0-132). The Hoehn-Yahr scale (0-5) was used for disease staging. The therapeutic medical regimen was recorded calculating the levodopa equivalent dose (LEDD) according to the method of Tomlinson et al [8]. As part of the preplanned investigations performed at baseline, and after 1 , 3 and 6 months of STN-DBS, autonomic symptoms were assessed with the Scopa-Aut questionnaire (0-69) [9], consisting of 26 items. It includes: gastrointestinal symptoms (7 items), urinary symptoms (6 items), cardiovascular symptoms (3 items), thermoregulation (4 items), pupillomotor function (1 item), sexual function (2 separate items for each gender) [9], each item is scored from 0 (never) to 3 (often), except for question 26, which is a yes/no question, and consequently not included in our statistical analysis. SCOPA-Aut score ranges from 0 to 69 , with higher scores expressing more severe symptoms. Anxiety and depression outcomes were assessed using the Hamilton Anxiety Rating Scale (HAMA) (14 parts), the HAMA ranges from 0 to 56 and the Hamilton Depression Rating Scale (HAMD) (24 parts), the HAMD ranges from 0 to 68 respectively, and quality of life was assessed using the 39-item Parkinson's Disease Questionnaire (PDQ-39), ranging from 0 to 124. Postoperative improvement rate (\%) was calculated as (preoperative score - postoperative score)/preoperative score $\times 100 \%$. Clinical assessments were performed at preoperative baseline (Med-OFF and Med-ON), 1 month after surgery (follow-up 1), 3 months after surgery (follow-up 2) and 6 months after surgery (follow-up 3).

\section{Surgical procedure}

Surgical procedures were carried out as our previously described [5].

\section{Stimulation programming}

One month after surgery, we turn on the stimulator and program the IPG [10], test the contacts on each electrode, and select the best stimulation target when the patient obtains satisfactory improvement with minimal side effects. After that, if necessary, the parameters can be adjusted through remote program control. First use the unipolar stimulation mode, the 
stimulation parameters: voltage $1.5 \llbracket 2.0 \mathrm{~V}$, frequency $130 \mathrm{~Hz}$, pulse width $60 \mathrm{~ms}$. Then gradually adjust the stimulation parameters until the best therapeutic effect is achieved.

\section{Statistical analyses}

All statistical analyses were performed using SPSS 25.0 (v25.0.0.0,SPSS Inc, Chicago/Illinois/USA). Continuous variables that followed, or approximately followed, a normal distribution are presented as mean \pm standard deviation( $(\mathrm{X} \pm \mathrm{s})$. Continuous variables that did not follow a normal distribution are presented as the median (M) and interquartile range (IQR). The Friedman test was used for continuous variables that did not follow a normal distribution and the KruskalWallis rank sum test was used for comparison between multiple groups. Correlation analysis method was used to analyze the factors influencing the improvement of autonomic dysfunction after DBS. The statistical significance threshold was fixed at $P<0.05$.

\section{Results}

\section{Patient population}

As mentioned in our previous article [5], the study group comprised 34 males and 23 females. The levodopa equivalent daily dose (LEDD) of the 57 patients preoperative was $(866.3 \pm 357.0)(125-1625) \mathrm{mg} / \mathrm{d}$, the preoperative Hoehn-Yahr stage was $(2.9 \pm 0.3)(2-4)$.

\section{Clinical outcomes}

In this study, 57 patients were included, operated, and examined preoperatively, with planned follow-up after 1,3 and 6 months of continuous STN-DBS. Comparisons between preoperative and postoperative (1, 3 and 6 months after surgery) clinical stages are summarized in Table 1 and Figure 1. At 6 months follow-up, the SCOPA-Aut scores (scales for outcomes in PD autonomic symptoms) [M (IQR)] improved significantly [14.59\% (18.32\%), 24.00\% (27.05\%), 22.16\% (27.07\%), respectively, all $P<0.001$ ] at 1 months, 3 months, 6 months of STN-DBS respectively. Analysis of the SCOPA-Aut subitems showed significant improvement only in urine and thermoregulation subitems at 6 months after operation $(P<0.001)$.

As mentioned in our previous article [5], UPDRS-III scores (Medication-off) were improved (55.42\%), the PDQ-39 scores were improved (47.39\%) and the LEDD had decreased by $40.08 \%$ at 6 months after surgery. The improvement rate of HAMA scores and HAMD scores of 57 patients was [41.7(34.9) \%;37.5(33.4) \% respectively (both $P<0.001)]$. 
Table 1

Comparison of preoperative and postoperative clinical state [M (IQR)]

\begin{tabular}{|c|c|c|c|c|c|c|c|c|c|}
\hline \multirow[t]{2}{*}{ Time } & \multirow[t]{2}{*}{ Preoperative } & \multicolumn{3}{|c|}{ Postoperative } & \multirow{2}{*}{$\begin{array}{l}\text { Total } \\
P\end{array}$} & \multirow[t]{2}{*}{$x^{2}$} & \multirow[t]{2}{*}{$P_{1}$} & \multirow[t]{2}{*}{$P_{2}$} & \multirow[t]{2}{*}{$P_{3}$} \\
\hline & & 1month & 3month & 6month & & & & & \\
\hline \multicolumn{10}{|l|}{ SCOPA-Aut } \\
\hline $\begin{array}{l}\text { SCOPA-Aut } \\
(0-69) \text { total }\end{array}$ & $22(12)$ & $18(14)$ & $18(10)$ & $16(12)$ & 0.003 & 5.399 & 0.001 & 0.001 & $<0.001$ \\
\hline $\begin{array}{l}\text { Gastrointestinal } \\
(\mathrm{Q} 1-7)\end{array}$ & $4(2.8)$ & $4(4)$ & $4(2.8)$ & $4(2)$ & 0.107 & 2.133 & 0.062 & 0.193 & 0.065 \\
\hline $\begin{array}{l}\text { Urinary } \\
\text { (Q8-13) }\end{array}$ & $7(4)$ & $4.5(3.8)$ & $4(5)$ & $4(5)$ & $<0.001$ & 11.062 & $<0.001$ & $<0.001$ & $<0.001$ \\
\hline $\begin{array}{l}\text { Cardiovascular } \\
\text { (Q14-16) }\end{array}$ & $2(1.8)$ & $2(1)$ & $2(1.7)$ & $2(2)$ & 0.248 & 1.418 & 0.049 & 0.107 & 0.345 \\
\hline $\begin{array}{l}\text { Thermoregulatory } \\
\text { (Q17-21) }\end{array}$ & $4(2)$ & $3(2.8)$ & $3(3)$ & $3(2.8)$ & $<0.001$ & 8.049 & $<0.001$ & $<0.001$ & $<0.001$ \\
\hline $\begin{array}{l}\text { Pupillomotor } \\
\text { (Q19) }\end{array}$ & $1(2)$ & $1(2)$ & $1(2)$ & $1(2)$ & 0.264 & 1.362 & 0.159 & 0.073 & 0.088 \\
\hline $\begin{array}{l}\text { Sexual } \\
(\mathrm{Q} 22-25)\end{array}$ & $2(1.7)$ & $2(2)$ & $2(1)$ & $2(1.7)$ & 0.792 & 0.346 & 0.659 & 0.871 & 0.542 \\
\hline $\begin{array}{l}\text { HAMA } \\
(0-56)\end{array}$ & $16(14)$ & $11(11)$ & $9(10)$ & $11(11)$ & $<0.001$ & 12.839 & $<0.001$ & $<0.001$ & $<0.001$ \\
\hline $\begin{array}{l}\text { HAMD } \\
(0-68)\end{array}$ & 14(13) & $9(9)$ & $9(8)$ & $9(9)$ & $<0.001$ & 11.664 & 0.004 & $<0.001$ & $<0.001$ \\
\hline $\begin{array}{l}P_{1}, P_{2} \text { and } P_{3} \text { value } \\
\text { months after surg } \\
\text { Rating Scale; } H A M\end{array}$ & SC, respectively & Scale $f$ & $\begin{array}{l}\text { f comp } \\
\text { utcome } \\
\text { Scale. }\end{array}$ & $\begin{array}{l}\text { ons bet } \\
\text { PD for }\end{array}$ & $\begin{array}{l}\text { en preo } \\
\text { onomi }\end{array}$ & $\begin{array}{l}\text { ative re } \\
\text { mpton }\end{array}$ & $\begin{array}{l}\text { s and } r \\
\text { HAMA: }\end{array}$ & $\begin{array}{l}\text { ults } 1,3 \\
\text { milton }\end{array}$ & $\begin{array}{l}\text { רd } 6 \\
\text { xiety }\end{array}$ \\
\hline
\end{tabular}

\section{The correlation analyses}

1. The correlation between the improvement rate of SCOPA-Aut scores, LEDD, PDQ-39 scores and the improvement rate of UPDRS- $₫$ scores (Med-off) (Table 2): There was no significant correlation between the improvement rate of SCOPA-Aut scores and the improvement rate of UPDRS- $\square$ scores (Med-off) ( $P>0.05)$. The reduction rate of LEDD was positively correlated with the improvement rate of UPDRS- $\square$ scores (Med-off) $(r=0.354, P=0.007)$, the improvement rate of PDQ-39 scores was positively correlated with the improvement rate of UPDRS- $\square$ scores $(r=0.461, P<0.001)$.

2. The correlation between the improvement rate of SCOPA-Aut scores and the improvement rate of PDQ-39 scores (Table 3): There was no significant correlation between the improvement rate of SCOPA-Aut scores and the improvement rate of PDQ-39 scores ( $P>0.05)$.

3. The correlation between SCOPA-Aut scores, PDQ-39 scores, and clinical variables (Table 4): In SCOPA-Aut scores there was no correlation with disease duration and Hoehn-Yahr grade $(P>0.05)$ and positively correlated with age $(r=0.428$, 
$P=0.001$ ), indicate that the older of the PD patients, the more serious of the autonomic dysfunction symptoms. In PDQ-39 scores there was positively correlated with disease duration $(r=0.296, P=0.025)$ and Hoehn-Yahr grade $(r=0.366, P=0.005)$. 4. The correlation between the improvement rate of SCOPA-Aut scores and the improvement rate of HAMA, HAMD scores (Table 5): The improvement rate of SCOPA-Aut scores was positively correlated with the improvement rate of HAMA scores $(r=0.325, P=0.015)$; The improvement rate of SCOPA-Aut scores was positively correlated with the improvement rate of HAMD scores $(r=0.265, P=0.049)$, indicate that the better the improvement rate of anxiety and depression, the better the improvement rate of autonomic dysfunction

Table 2 The correlation between SCOPA-Aut scores, LEDD, PDQ-39 scores and motor symptoms improvement rate 6 months after surgery

\begin{tabular}{|lllll|}
\hline & improvement rate (\%) & the improvement rate of UPDRS- $\mathbb{Z}$ scores (Med-off ) (\%) & r & $\boldsymbol{P}$ \\
\hline SCOPA- Aut & 22.16 & 55.42 & 0.086 & 0.527 \\
\hline LEDD & 40.08 & 55.42 & 0.354 & 0.007 \\
\hline PDQ-39 & 47.39 & 55.42 & 0.461 & $<0.001$ \\
\hline
\end{tabular}

Table 3 The correlation between the improvement rate of SCOPA-Aut scores and the improvement rate of PDQ-39 scores 6 months after surgery

\begin{tabular}{|lllll|}
\hline & improvement rate (\%) & the improvement rate of PDQ-39 scores (\%) & $\mathbf{r}$ & $\boldsymbol{P}$ \\
\hline SCOPA- Aut & 22.16 & 47.39 & 0.126 & 0.355 \\
\hline
\end{tabular}

Table 4 The correlation between SCOPA-Aut scores, PDQ-39 scores and clinical variables 6 months after surgery

\begin{tabular}{|lllllll|}
\hline & Age & \multicolumn{4}{c|}{ Disease duration } & \multicolumn{2}{l|}{ Hoehn-Yahr grade } \\
\cline { 2 - 7 } & $\mathbf{r}$ & $\mathbf{P}$ & $\mathbf{r}$ & $\mathbf{P}$ & $\mathbf{r}$ & $\mathbf{P}$ \\
\hline SCOPA-Aut & 0.428 & $\mathbf{0 . 0 0 1}$ & 0.221 & 0.101 & 0.086 & 0.528 \\
\hline PDQ-39 & $\mathbf{0 . 0 0 6}$ & 0.967 & 0.296 & $\mathbf{0 . 0 2 5}$ & 0.366 & $\mathbf{0 . 0 0 5}$ \\
\hline
\end{tabular}

Table 5 The correlation between the improvement rate of SCOPA-Aut scores, and the improvement rate of HAMA, HAMD scores 6 months after surgery

\begin{tabular}{|llllll|}
\hline & improvement rate (\%) & the improvement rate of SCOPA-Aut scores (\%) & $\mathbf{r}$ & $\boldsymbol{P}$ \\
\hline HAMA & 34.27 & 22.16 & 0.325 & $\mathbf{0 . 0 1 5}$ \\
\hline HAMD & 26.95 & 22.16 & 0.265 & $\mathbf{0 . 0 4 9}$ \\
\hline
\end{tabular}

\section{Discussion}


DBS is widely used in the clinical treatment of PD because of its minimally invasive, adjustable, reversible. Most PD patients experience autonomic dysfunction at different clinical stages, the incidence rate is $14 \%-80 \%[11,12]$. Autonomic dysfunction in PD includes gastrointestinal malfunction (constipation, dysphagia, or choking); urinary disturbance (increased nocturia, frequent urination, and endless urination); sexual dysfunction (impotence, vaginal dryness, etc); thermoregulatory aberrance (sweat, intolerance, etc); cardiovascular dysregulation (postural hypotension and dizziness), pupillo-motor and tear abnormalities [13], which seriously affects the quality of life in PD patients. In this retrospective study of 57 PD patients treated with STN-DBS, through correlation analysis, it was found that the older the patients with Parkinson's disease, the more serious the autonomic dysfunction. It is speculated that the older age of onset of Lewy bodies is related to the deposition of parts related to autonomic dysfunction.

The mechanism for the improvement of the symptoms of autonomic dysfunction may be that STN-DBS directly regulates the basal ganglia-thalamus-cortical circuit, thereby regulating the thalamus, lateral frontal lobe, and anterior cingulate gyrus (the center of the autonomic nervous system) to improve the symptoms of autonomic dysfunction [14]. The effect of STN-DBS on gastrointestinal dysfunction works by improving constipation, dysphagia and salivation [15]. Studies have shown that the effect of STN-DBS on urinary disturbance works by reducing the detrusor muscle tension, increasing bladder capacity and reflexing volume [16]. The severity of bladder dysfunction seems to be associated with the relative degeneration of the caudate nucleus, amongst other areas [17]. STN-DBS can improve the temperature perception of PD patients, DBS can improve hyperhidrosis and heat intolerance [18], which may be related to the stimulation of the tail of STN, the ventral thalamus and the zona incerta (ZI). The effect of STN-DBS on sexual dysfunction may be related to stimulation of the medial preoptic nucleus, anterior hypothalamic nucleus, and nucleus accumbens, resulting in changes in its activity [19]. The effect of STN-DBS on cardiovascular dysfunction works by increasing heart rate, the sensitivity of baroreceptors and peripheral vascular tone, it can improve postural hypotension in PD patients [20], which may be related to stimulating the Limbic of STN or the zona incerta (ZI) [21]. Frontal cortex, cingulate cortex, insula, thalami, basal ganglia and periaqueductal grey matter may be relevant with gastrointestinal function [22]. STN-DBS can activate the nerve fibres projecting from hypothalamus and crossing the subthalamic nucleus, that might be affected gastrointestinal function. Previous studies have shown that STN-DBS can improve autonomic dysfunction in PD patients [23-27]. Few previous reports have used SCOPA-Aut to assess the effect of STN-DBS on autonomic dysfunction. Previous studies have used NMSQ to assess NMS [28], However, this scale is only for NMSS, not for autonomic dysfunction specifically. The improvement of dysautonomic fluctuations after chronic stimulation was remarkable especially for some symptoms. Urinary dysfunction showed the greatest reduction in the number of symptoms reported by patients after the surgery. Regarding autonomic symptoms, the significant improvement of Scopa-Aut total score after 6 months of STN-DBS, the SCOPA-Aut total score were increased by $22.16 \%(P<0.001)$ after 6 months of follow-up, indicating that STN-DBS has an improvement effect on autonomic dysfunction of Parkinson's disease in the short term. However, the results were not equal to all categories of symptoms. Analyses of each SCOPA-Aut domain showed significant improvement at 6 months only for the Urinary and thermoregulatory dysfunction showed a remarkable decrease after the chronic stimulation. However, one study of 24 patients reported similar results as presented here, where Scopa-Aut improved after 3 months of follow-up but with subsequent deterioration [29-31]. So We need further follow-up studies to confirm this, a prospective study with pre-operative and post-operative urodynamics would yield more detailed information about the effect of STNDBS on bladder function. We could not predict the preoperative impact on SCOPA-Aut, but the parallel improvement of PDQ-39 scores were identified as a significant covariate. This might imply that the degree of improvement of autonomic dysfunction plays an important role in improving quality of life. However there was no significant correlation between the improvement rate of SCOPA-Aut scores and the improvement rate of PDQ-39 scores ( $P>0.05)$. May be due to so many factors of the STN-DBS on quality of life, in addition to the motor symptoms associated with important role, the NMS impact is very important also, not just the autonomic dysfunction, such as anxiety, depression, numbness, pain, sleep disorders, cognitive impairment, hallucinations and other influence is very significant also, so we need to consider a lot of factors comprehensively, judge comprehensively. 
Previous reports SCOPA-Aut scores increase by age and disease duration [32]. In our study, SCOPA-Aut total scores increase by age, it indicates that the autonomic dysfunction will gradually worsen with the age.

Autonomic dysfunction is related to emotional disorders, and there may be a common mechanism [33]. The 5-HT neurotransmitter system in patients with Parkinson's disease may be one of the mechanisms related to autonomic dysfunction and emotional disorders. In our study, the improvement rate of SCOPA-Aut scores was positively correlated with the improvement rate of HAMA scores $(r=0.325, P=0.015)$; The improvement rate of SCOPA-Aut scores was positively correlated with the improvement rate of HAMD scores $(r=0.265, P=0.049)$, indicate with the improvement of anxiety and depression symptoms in Parkinson's disease, autonomic dysfunction will also improve.

Quality of autonomic dysfunction deserve more focus both in the preoperative and postoperative evaluation of PD patients for STN-DBS. Future studies should consider including this factor among the main outcomes, especially in studies focusing on optimal electrode location and tissue activated (VTA) in STN, closed-loop DBS, directional electrode, functional brain imaging and brain networks.

\section{Limitations:}

There are also some deficiencies in this study :(1) the sample is small and it was retrospective study. (2) SCOPA-Aut scores for preoperative evaluation of autonomic dysfunction are subjective. (3) Postoperative follow-up time was 6 months, and the time is short.

\section{Conclusion}

STN-DBS can improve autonomic dysfunction of PD, urinary and thermoregulatory aspects of autonomic dysfunction were improved in the short term postoperatively. There was a close relationship between improved autonomic dysfunction and improved anxiety and depression 6 months. We should pay more attention to the autonomic dysfunction in Parkinson's disease, detailed preoperative evaluation and postoperative follow-up, so as to better improve the QOL of patients.

\section{Abbreviations}

PD: Parkinson's disease

STN: Subthalamic nucleus

DBS: Deep brain stimulation

UPDRS-『: Unified Parkinson's Disease Rating Scale-Part III

LEDD: Levodopa equivalent day dose

QOL: Quality of life

PDQ-39: 39-Item Parkinson's Disease Questionnaire

NMS : non-motor symptoms

SCOPA-Aut : the Scales for Outcomes in Parkinson's Disease-Autonomic questionnaire

HAMA : the Hamilton Anxiety Rating Scale

Page 8/13 
HAMD : the Hamilton Depression Rating Scale

LCT: Levodopa challenge test

Med-off: Medication-off

Med-on: Medication-on

IPG: Implantable pulse generator

\section{Declarations}

\section{Ethics approval and consent to participate}

All authors clearly consented to and approved this manuscript. The study strictly followed the ethical principles set out in the "Declaration of Helsinki ". All patients and their families (spouse or children) have informed consent and signed an informed consent form, the study was approved by the Medical Ethics Committee of the First Hospital of Hebei Medical University and the Medical Ethics Committee of Beijing Tiantan Hospital affiliated to Capital Medical University, and the ethics committee approved this form of proxy consent.

\section{Consent for publication}

Not applicable.

\section{Availability of data and materials}

The datasets used and/or analyzed during the current study not publicly available due to privacy reasons of patients, but are available from the corresponding author on reasonable request.

\section{Competing interests}

There are no competing interests.

\section{Funding}

This study was funded by S\&T Program of Hebei (21377736D), Beijing Municipal Administration of Hospitals Clinical Medicine Development of Special Funding Support (XMLX201833, ZYLX201820), National Natural Science Foundation of China (81971070). The funder had no role in the study design, data collection, or analysis, the decision to publish or the preparation of the manuscript.

\section{Author information}

\section{Affiliations}

Department of neurosurgery, the First Hospital of Hebei Medical University, Shijiazhuang, Hebei, 050031, China

Feng Zhang, Cong-Hui Li, Ji-Wei Wang, Yu-Jing Xing \& Chen Yang

Departments of Neurosurgery, The First Affiliated Hospital, Zhejiang University School of Medicine, Hangzhou, Zhejiang, 310000, China

Feng Wang 
Departments of Neurosurgery, General Hospital of Ningxia Medical University, Yinchuan, Ningxia, 750004, China

Feng Wang

Department of Neurosurgery, Beijing Tiantan Hospital, Capital Medical University, Beijing, 100070, China

Chun-Lei Han, Shi-Ying Fan, Dong-Mei Gao, Jianguo Zhang \& Fangang Meng

Beijing Neurosurgical Institute, Capital Medical University, Beijing, 100070, China

Fangang Meng

Beijing Key Laboratory of Neurostimulation, Beijing, 100070, China

Fangang Meng

Chinese Institute for Brain Research, Beijing, 102206, China

Fangang Meng

\section{Authors' Contributions}

Study concept and design: F Z, FG M. Data collection: F Z, SY F, CL H, DM G, CH L, JW W, YJ X, C Y. Analysis and interpretation: F Z, F W, CH L, JW W. Drafting of the manuscript: F Z. Critical revision of the manuscript: F Z, FG M. Study supervision: $F Z$, FG M. F Z was a major contributor in writing the manuscript. The authors read and approved the manuscript.

\section{Corresponding author}

Correspondence to Fangang Meng.

\section{Acknowledgements}

We are grateful to all the patients recruited for this study. We thank the Medical Ethics Committee of the First Hospital of Hebei Medical University and the medical ethics committee of IRB of Beijing Tiantan Hospital Affiliated to Capital Medical University for the approval of our study.

\section{References}

1. Kalia LV, Lang AE. Parkinson's disease. Lancet,2015,386:896-912.DOI:10.1016/S0140-6736(14)61393-3.

2. Wichmann T, Delong MR. Deep brain stimulation for movement disorders of basal ganlia origin: restoring function or functionslity? [J]. Neutotherapeutics, 2016, 13: 264-283. DOI:10.1007/s13311-016-0426-6.

3. Halim A, Baumgartner L, Binder DK. Effect of deep brain stimulation on autonomic dysfunction in patients with Parkinson's disease. J Clin Neurosci. 2011,18(6):804-6. DOI:10.1016/j.jocn.2010.10.015.

4. Pietraszko W, Furgala A, Gorecka-Mazur A,et al. Efficacy of deep brain stimulation of the subthalamic nucleus on autonomic dysfunction in patients with Parkinson's disease. Folia Med Cracov. 2013,53(2):15-22.

5. Zhang F, Wang F, Li W, et al. Relationship between electrode position of deep brain stimulation and motor symptoms of Parkinson's disease. BMC Neurol,2021,21(1):122. DOI:10.1186/s12883-021-02148-1.

6. Parkinson's Disease and Dyskinesia Group, Neurology Branch, Chinese Medical Association, Professional Committee of Parkinson's Disease and Dyskinesia, Neurologist Branch. Chinese Medical Association. Diagnostic criteria for 
Parkinson's disease in China (2016 edition) [J]. Chinese Journal of Neurology, 2016, 49(4): 268-271.

DOI:10.3760/cma.j.issn.1006-7876.2016.04.002.

7. Zach H, Walter U, Liepelt-Scarfone I, et al. Diagnostics of clinical and prodromal idiopathic Parkinson's disease:New criteria.Nervenarzt, 2017, 88(4):356-364. DOI:10.1007/s00115-017-0290-6.

8. Tomlinson CL, Stowe R, Patel S, et al. Systematic review of levodopa dose equivalency reporting in Parkinson's disease. Mov Disord, 2010, 25(15): 2649-53. DOI:10.1002/mds.23429.

9. Visser M, Marinus J, Stiggelbout AM, et al. Assessment of autonomic dysfunction in Parkinson's disease: the ScopaAut. Mov Disord, 2004, 19(11):1306-12. DOI: 10.1002/mds.20153.

10. Horn A, Reich M, Vorwerk J, et al. Connectivity Predicts deep brain stimulation outcome in Parkinson disease. Ann Neurol, 2017,82(1):67-78. DOI: 10.1002/ana.24974.

11. Sjodahl HC, Hagell P, Nilsson MH.Motor and non-motor predictors of illness-related distress in Parkinson's disease [J]. Parkinsonism Relat Disord, 2012, 18(3): 299-302. DOI:10.1016/j.parkreldis.2011.10.015.

12. Martinez-Martin P, Valldeoriola F, Tolosa E, et al. Bilateral subthalamic nucleus stimulation and quality of life in advanced Parkinson's disease. Mov Disord, 2002, 17:372-77. DOI:10.1002/mds.10044.

13. Zhang Z, Chen SD. Research progress of autonomic dysfunction in Parkinson's disease. Chin J Contemp Neurol Neurosurg, 2019, 19(5): 367-373. DOI:10.3969/j.issn.1672ه6731.2019.05.012.

14. Trachani E, Constantoyannis C, Sirrou V, et al. Effects of subthalamic nucleus deep brain stimulation on sweating function in Parkinson's disease. Clin Neurol Neurosurg, 2010, 112(3):213-217. DOI:10.1016/j.clineuro.2009.11.015.

15. Lengerer S, Kipping J, Rommel N, et al. Deep-brain-stimulation does not impair deglutition in Parkinson's disease. Parkinsonism Relat Disord, 2012, 18(7):847-53. DOI:10.1016/j.parkreldis.2012.04.014.

16. Herzog J, Weiss PH, Assmus A, et al. Improved sensory gating of urinary bladder afferents in Parkinson's disease following subthalamic stimulation. Brain, 2008, 131:132-45. DOI:10.1093/brain/awm254.

17. Fowler CJ, Dalton C, Panicker JN. Review of neurologic diseases for the urologist. Urol Clin North Am, 2010, 37(4): 517-26. DOI: 10.1016/j.ucl.2010.07.004.

18. Witjas T, Kaphan E, Regis J, et al. Effects of chronic subthalamic stimulation on nonmotor fluctuations in Parkinson's disease. Mov Disord, 2007, 22:1729-34. DOl:10.1002/mds.21602.

19. Wang XH, Lin Zhang, Wang SJ, et al. Effects of deep brain stimulation on non-motor symptoms of Parkinson's disease. J Clin Neurol, 2018, 31(2):147-150.

20. Liu KD, Shan DE, Kuo TB, et al. The effects of bilateral stimulation of the subthalamic nucleus on heart rate variability in patients with Parkinson's disease. J Neurol, 2013, 260(7):1714-23. DOI: 10.1007/s00415-013-6849-7.

21. Priori A, Cinnante C,Genitrini S,et al. Non-motor effects of deep brain stimulation of the subthalamic nucleus in Parkinson's disease: preliminary physiological results. Neurol Sci, 2001, 22(1): 85-6. DOI:10.1007/s100720170059.

22. Vandenbergh J, Dupont P, Fischler B, et al. Regional brain activation during proximal stomach distention in humans: $A$ positron emission tomography study. Gastroenterology, 2005, 128(3): 564-73. DOI: 10.1053/j.gastro.2004.11.054.

23. Halim A, Baumgartner L, Binder DK. Effect of deep brain stimulation on autonomic dysfunction in patients with Parkinson's disease. J Clin Neurosc, 2011, 18: 804-806. DOI: 10.1016/j.jocn.2010.10.015.

24. Herzog J, Weiss PH, Assmus A, et al. Subthalamic stimulation modulates cortical control of urinary blader in Parkinson's disease. Brain, 2006, 129: 3366-3375. DOI: 10.1093/brain/awl302. Epub 2006 Oct 31.

25. Trachani E, Constantoyannis C, Sirrou V, et al. Effects of subthalamic nucleus stimulation on sweating function in Parkinson's disease. Clinical Neurology Neurosurg, 2012, 112: 213-217. DOI: 10.1016/j.clineuro.2009.11.015. Epub 2009 Dec 22.

26. Nazzaro JM, Pahwa R, Lyons KE. The impact of bilateral subthalamic stimulation on non-motor symptoms of Parkinson's disease. Parkinsonism Rel Disord, 2011, 17: 606-609. DOI: 10.1016/j.parkreldis.2011.05.009. Epub 2011 
Jun 12.

27. Arai E, Arai M, Uchiyama T, et al. Subthalamic deep brain stimulation can improve gastric emptying in Parkinson's disease. Brain, 2012,135:1478-1485. DOI: 10.1093/brain/aws086. Epub 2012 Apr 19.

28. Chaudhuri KR, Martinez-Martin P, Anthony HV, et al. International multicenter pilot study of the first comprehensive self-completed nonmotor symptoms questionnaire for Parkinson's disease: the NMSQuest study, Mov Disord,2006, 21(7), 916-923. DOI: 10.1002/mds.20844.

29. Kurcova S, Bardon J, Vastik M, et al. Bilateral subthalamic deep brain stimulation initial impact on nonmotor and motor symptoms in Parkinson's disease: an open prospective single institution study. Medicine (Baltimore), 2018,97(5): e9750. DOI: 10.1097/MD.0000000000009750.

30. Bjerknes S, Skogseid IM, Hauge TJ, et al. Subthalamic deep brain stimulation improves sleep and excessive sweating in Parkinson's disease. NPJ Parkinsons Dis, 2020,14, 6:29. DOI: 10.1038/s41531-020-00131-0.

31. Dafsari HS, Silverdale M, Strack M, et al. Nonmotor symptoms evolution during 24 months of bilateral subthalamic stimulation in Parkinson's disease. Mov. Disord,2018, 33(30), 421-430. DOI: 10.1002/mds.27283. Epub 2018 Feb 21.

32. Arnao V, Cinturino A, Valentino F, et al. In patient's with Parkinson disease, autonomic symptoms are frequent and associated with other non-motor symptoms. Clin. Auton Res,2015, 25(5): 301-307. DOI: 10.1007/s10286-015-0306-x. Epub 2015 Sep 10.

33. Cao CJ, Yu SY, Zuo LJ, et al. Study on the correlation of autonomic dysfunction with motor symptoms and non-motor symptoms in patients with Parkinson's disease. Chin J Clinicians (Electronic Edition), 2013, 7(15): 6898-6903. DOI: 10.3877/cma.j.issn.1674-0785.2013.15.028.

\section{Figures}



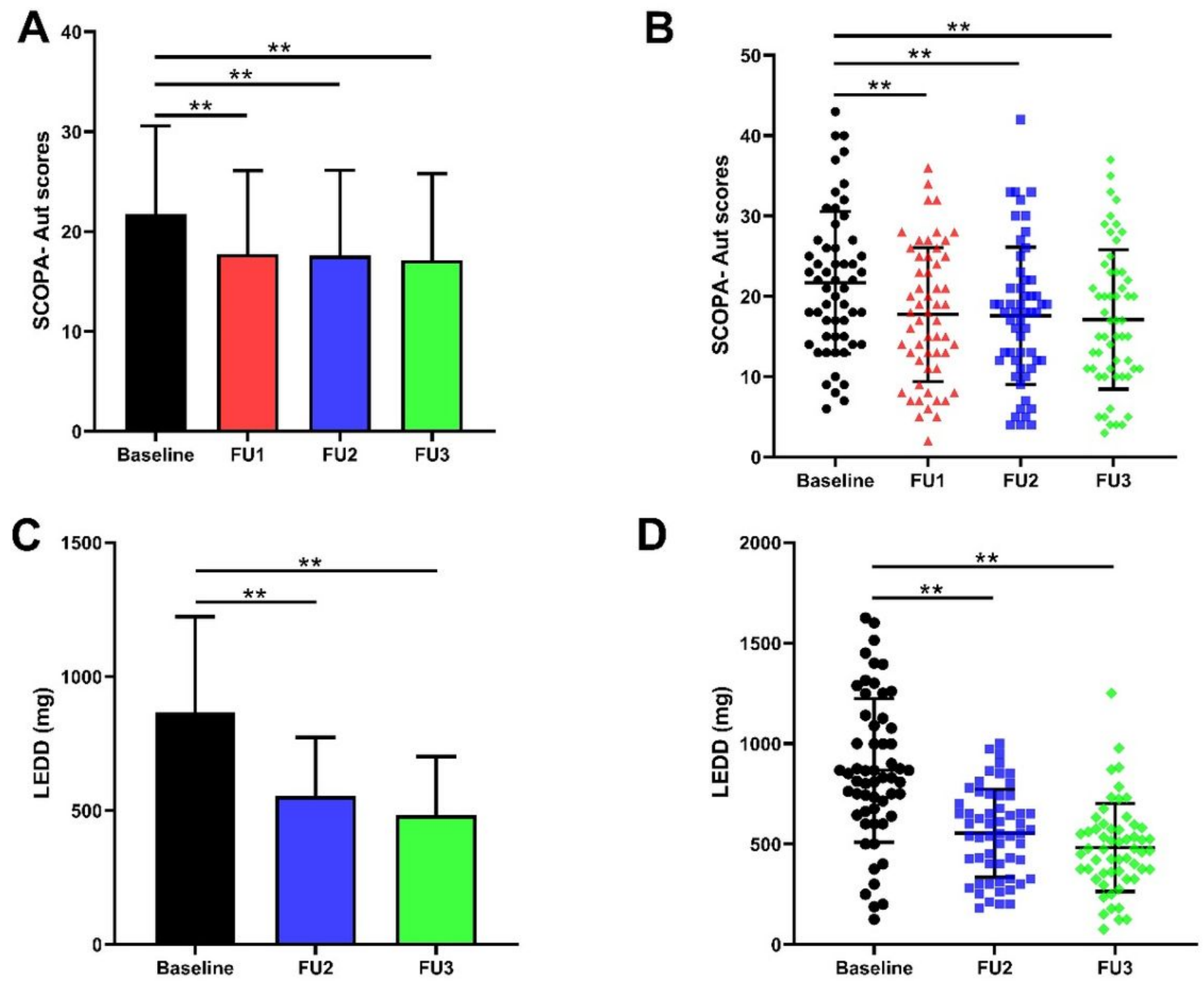

Figure 1

SCOPA- Aut and LEDD comparison between pre-and postoperative clinical state. (A-B) SCOPA- Aut scores were improved

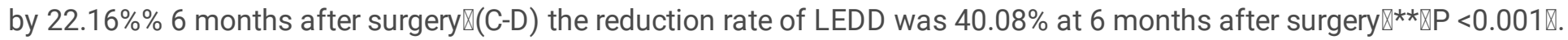
(STN-DBS: subthalamic nucleus - deep brain stimulation》 SCOPA-Aut: the Scale for Outcomes in PD for Autonomic Symptoms \LEDD: Levodopa equivalent dose) [Baseline: baseline; FU1: 1 month after surgery; FU2: 3 months after surgery; FU3: 6 months after surgery] 